\title{
A HÖRMANDER TYPE CRITERION FOR QUASI-RADIAL FOURIER MULTIPLIERS
}

\author{
HENRY DAPPA AND HAJO LUERS
}

\begin{abstract}
We state practicable sufficient conditions on quasi-radial functions $m \circ \rho(\xi)=m(\rho(\xi))$ to be Fourier multipliers in $L^{p}\left(\mathbf{R}^{n}\right)$. Here $m$ is a bounded function and $\rho$ is a homogeneous distance function. The conditions on $m$ are given in terms of localized Bessel potentials and those on $\rho$ reflect and generalize basic properties of the norm in $\mathbf{R}^{\prime \prime}$. The results are related to those of Madych [7] and Fabes and Rivière [3] and improve their results (specialized to quasi-radial multipliers). The proof utilizes Madych's approach [7] and interpolation properties of localized Bessel potential spaces [2].
\end{abstract}

0. A function $m \in L^{\infty}\left(\mathbf{R}^{n}\right)$ is called a Fourier multiplier in $L^{p}\left(\mathbf{R}^{n}\right), m \in M_{p}\left(\mathbf{R}^{n}\right)$, if the relation $(T f)^{\wedge}(\xi)=m(\xi) \hat{f}(\xi)$ defines a continuous endomorphism on $L^{p}\left(\mathbf{R}^{n}\right)$ where $f^{\wedge}$ denotes the Fourier transform of $f \in L^{p} \cap L^{2}\left(\mathbf{R}^{n}\right)$; the multiplier norm $\|m\|_{M_{n}}$ equals the operator norm of $T$. For the general definition of localized Bessel potential spaces $S(q, \gamma)$ we refer to Connett and Schwartz [2]. In case $\gamma>1 / q$, $1<q<\infty$, these spaces can be identified with the spaces of functions of weak bounded variation, as was shown by Gasper and Trebels [5]. If $\gamma \in \mathbf{N}, 1 \leqslant q \leqslant \infty$, then $\mathrm{WBV}_{q, \gamma}=\left\{m \in L^{\infty}(0, \infty): m, m^{\prime}, \ldots, m^{(\gamma-1)}\right.$ are locally absolutely continuous and $\|m\|_{q, \gamma}=\|m\|_{\infty}+\sup _{R>0}\left(\int_{R}^{2 R}\left|t^{\gamma} m^{(\gamma)}(t)\right|^{q} d t / t\right)^{1 / q}<\infty$ if $q<\infty$ or $\|m\|_{q, \gamma}$

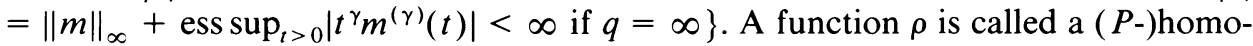
geneous distance function if $\rho$ is continuous on $\mathbf{R}^{n}$, positive on $\mathbf{R}_{0}^{n}=\mathbf{R}^{n} \backslash\{0\}$ and satisfies $\rho\left(t^{P} x\right)=t \rho(x)$ for $t>0$ and $x \in \mathbf{R}^{n}$. Here $P$ is a fixed real $(n \times n)$-matrix with eigenvalues having positive real parts.

Examples are given in $[\mathbf{1}, 10$ and 14]; further examples are:

$$
\begin{gathered}
\rho_{1}(x)=x_{1}^{6}-\sin ^{2}\left(x_{1}^{3} / x_{2}\right) x_{1}^{3} x_{2}+x_{2}^{2}, \quad \rho_{2}(x)=\sum_{j=1}^{n}\left|x_{j}\right|^{\alpha_{j}} \quad\left(\alpha_{j}>0\right), \\
\rho_{3}(x)=\left(x_{1}^{4}+x_{2}^{2}\right)^{1 / 2}+\left|x_{1}\right|^{4 / 5}\left|x_{2}\right|^{8 / 5} /\left(x_{1}^{2}+\left|x_{2}\right|\right) .
\end{gathered}
$$

Throughout the paper $c$ and $C$ denote generic constants, $N$ the least integer greater than $n / 2$, and $t_{+}=\max \{0, t\}$.

THEOREM 1. Let $\rho \in C^{N}\left(\mathbf{R}_{0}^{n}\right)$ be a homogeneous distance function, $1<p<\infty$, and $m \in \mathrm{WBV}_{q, \gamma}$, where $1 \leqslant q \leqslant \infty$ and $\gamma>n|1 / p-1 / 2|+(1 / q-|1 / p-1 / 2|)_{+}$. Then $m \circ \rho \in M_{p}\left(\mathbf{R}^{n}\right)$ and $\|m \circ \rho\|_{M_{p}} \leqslant C\|m\|_{q, \gamma}$ with $C$ independent of $m$.

Received by the editors September 6, 1983.

1980 Mathematics Subject Classification. Primary 42B15, 42B25.

Key nords and phrases. Fourier multipliers, Hörmander criterion. 
Peral and Torchinsky [9] have shown: if $0<p<\infty, 1<q \leqslant \infty$ and $m \in \mathrm{WBV}_{q, \gamma}$ for some integer $\gamma>\nu|1 / p-1 / 2|+1 / q$, then $m \circ \rho$ is a multiplier in $H^{p}\left(\mathbf{R}^{n}\right)$. Here $\rho \in C^{\infty}\left(\mathbf{R}_{0}^{n}\right)$ is the $P$-homogeneous distance function determined by $\left|\rho(x)^{-P} x\right|=1$ if $x \neq 0$. The matrix $P$ has to satisfy $t^{\alpha}|x| \leqslant\left|t^{P} x\right| \leqslant t^{\beta}|x|$ for some $1 \leqslant \alpha \leqslant \beta$ and all $t \geqslant 1, x \in \mathbf{R}^{n}$. $\nu$ is the trace of $P$.

Obviously Theorem 1 is an improvement of Peral and Torchinsky's result in case $1<p<\infty$ as the following examples show:

$(1-\rho(\xi))_{+}^{\lambda} \in M_{p}\left(\mathbf{R}^{n}\right)$ if $\lambda>(n-1)|1 / p-1 / 2|$ and $1<p<\infty$. This multiplier may be of interest for the generalized Riesz summation of inverse Fourier integrals. Analogous results were

(i) obtained by Löfström [6] and Peetre [8] for a class of distance functions $\rho$ satisfying $\rho \in C^{\infty}\left(\mathbf{R}_{0}^{n}\right)$ and $\rho(t x)=t^{\alpha} \rho(x)$ for some $\alpha>0$ and all $t>0, x \in \mathbf{R}^{n}$. See also the discussion of the above multiplier in the radial case in Fefferman [4].

$e^{i \rho(\xi)}(1+\rho(\xi))^{-\beta} \in M_{p}\left(\mathbf{R}^{n}\right)$ if $\beta>n|1 / p-1 / 2|$ and $1<p<\infty$.

(ii) This result extends that of Sjöstrand [11] from the radial to the quasi-radial case.

For the proof of Theorem 1 the approach via Littlewood-Paley functions is used. We employ the same Littlewood-Paley functions as Madych [7]:

$$
\begin{aligned}
& g_{1}(f)(x)=\left(\int_{0}^{\infty}\left|K_{t} * K_{t} * f(x)\right|^{2} d t / t\right)^{1 / 2}, \\
& g_{2}(f)(x)=\left(\int_{0}^{\infty} \int t^{-\nu}\left(1+\left|t^{-P^{*}}(x-y)\right|^{\gamma}\right)^{-2}\left|K_{t} * f(y)\right|^{2} d y d t / t\right)^{1 / 2},
\end{aligned}
$$

where $K_{t}=F^{-1}[\varphi(t r(\xi))], \varphi \in C^{\infty}(\mathbf{R})$ is a nonnegative bump function supported in [1, 2], $r \in C^{\infty}\left(\mathbf{R}_{0}^{n}\right)$ is a fixed $P$-homogeneous distance function (see [14, p. 1255]), $F^{-1}$ denotes the inverse Fourier transformation, $P^{*}$ the transpose of $P, \nu=\operatorname{trace}(P)$, $f \in L^{2}\left(\mathbf{R}^{n}\right)$ and $\gamma>n / 2$.

To be precise we point out that the matrix $P$ is more general than that of Madych [7] which is assumed to be "good" or at least "reasonable", but this does not affect the $L^{p}$-behaviour of the $g$-functions.

The approach via Littlewood-Paley functions first occurs in Stein [12] and is generalized by Madych [7] to obtain an anisotropic version (Proposition 4) of the Hörmander multiplier criterion [13]. Madych's assumption on the multiplier $m$ can be expressed by

$$
\left.\left.\sup _{t>0} \int|| x\right|^{\gamma} F^{-1}\left[\varphi(r(\xi)) m\left(t^{P} \xi\right)\right](x)\right|^{2} d x \leqslant B^{2}<\infty, \quad \gamma>n / 2 .
$$

The following lemma presents a practicable sufficient condition for this assumption to be satisfied in the quasi-radial case. 
Lemma 1. Let $m \in C^{\infty}(0, \infty)$ be compactly supported in $(0, \infty)$ and let $\rho$ be as in Theorem 1. Then for every $\gamma>n / 2$

$$
\left.\left.\int|| x\right|^{\gamma} F^{-1}[\varphi(r(\xi)) m(t \rho(\xi))](x)\right|^{2} d x \leqslant c\|m\|_{2, \gamma}^{2}
$$

with $c$ independent of $m$ and $t>0$.

Madych's method now yields

Lemma 2. Let $m, \rho$, and $\gamma$ be as in Lemma 1. Then $\|m \circ \rho\|_{M_{p}} \leqslant c\|m\|_{2, \gamma}$ for every $1<p<\infty$, where $c$ is independent of $m$.

A combination of Lemma 2 with interpolation and embedding arguments of Connett and Schwartz [2] on localized Bessel potentials will lead to Theorem 1.

1.

Proof of Lemma 1. (a) The case $\gamma=k \in \mathbf{N}$. We omit the region of integration if it is the whole $\mathbf{R}^{n} . \sigma \in \mathbf{N}^{n}$ is a multi-index, $|\sigma|=\sigma_{1}+\cdots+\sigma_{n}, x^{\sigma}=x_{1}^{\sigma_{1}} \cdots x_{n}^{\sigma_{n}}$, $D^{\sigma}=(\partial / \partial x)^{\sigma},(\partial / \partial x)^{\sigma}=\left(\partial / \partial x_{1}\right)^{\sigma_{1}} \cdots\left(\partial / \partial x_{n}\right)^{\sigma_{n}}$. By Plancherel's theorem and simple estimates,

$$
\begin{aligned}
I_{k} & =\left.\left.\int|| x\right|^{k} F^{-1}[m(R \rho(\cdot)) \varphi(r(\cdot))](x)\right|^{2} d x \\
& \leqslant c \sum_{|\sigma|=k} \int\left|\left(\frac{\partial}{\partial \xi}\right)^{\sigma}[m(R \rho(\xi)) \varphi(r(\xi))]\right|^{2} d \xi \\
& \leqslant c \sum_{|\sigma| \leqslant k} \int_{1 \leqslant r(\xi) \leqslant 2}\left|\left(\frac{\partial}{\partial \xi}\right)^{\sigma}[m(R \rho(\xi))]\right|^{2} d \xi .
\end{aligned}
$$

It is an immediate consequence of $\rho$ and $r$ being $P$-homogeneous distance functions that there are $c_{1}, c_{2}>0$ such that $c_{1} r(\xi) \leqslant \rho(\xi) \leqslant c_{2} r(\xi)$. Hence, the domain of integration is contained in $\left\{\xi: c_{1} \leqslant \rho(\xi) \leqslant 2 c_{2}\right\}$ and we may estimate the derivatives and arbitrary powers of $\rho$ from above and below.

Applying the chain rule and converting to Riemann-Stieltjes integrals we get

$$
\begin{aligned}
I_{k} & \leqslant c \sum_{l=0}^{k} \int_{c_{1} \leqslant \rho(\xi) \leqslant 2 c_{2}}\left|(R \rho(\xi))^{\prime} m^{(l)}(R \rho(\xi))\right|^{2} \rho(\xi)^{-\nu} d \xi \\
& \leqslant c \sum_{l=0}^{k} \int_{c_{1} R}^{2 c_{2} R}\left|t^{\prime} m^{(l)}(t)\right|^{2} d t / t \leqslant c\|m\|_{2, k}^{2}
\end{aligned}
$$

since we can cover $\left[c_{1} R, 2 c_{2} R\right]$ with a fixed number of intervals $\left[2^{j}, 2^{j+1}\right]$ and $\mathrm{WBV}_{2, l} \subset \mathrm{WBV}_{2, k}$ for $l \geqslant k$.

(b) The case $\gamma=k+\delta, 0<\delta<1$. This case can be treated by the argument of [2] on pp. 70-71. Hence, we only point out the important steps. Let $S(q, \alpha)$ be defined as a $V^{*}$-localization of the Bessel potential space $L_{\alpha}^{q}$, and $S_{0}(q, \alpha)$ as a $W^{*}$-localization. For the definitions we refer to [2, $\$ 1.3$ and 2.4$]$. 
Let the space $\mathscr{F}=\mathscr{F}\left(S_{0}(2, k), S_{0}(2, k+1)\right)$ of functions on $\bar{S}=\{z \in \mathbf{C}: 0 \leqslant$ $\operatorname{Re} z \leqslant 1\}$ be defined as in [2, §1.4]. In order to smooth functions in $S_{0}(q, \alpha)$ take $\eta \in C^{\infty}(0, \infty)$ with $\operatorname{supp} \eta=[1 / 2,2], \eta \geqslant 0$ and $\int_{0}^{\infty} \eta(t) d t / t=1$, set $\eta_{\varepsilon}(t):=$ $\varepsilon \eta\left(t^{\varepsilon}\right)$ for $\varepsilon>0$ and define for any $f \in \mathscr{F}$ with $f(\delta)=m$

$$
f_{\varepsilon}(z, t):=\int_{0}^{\infty} \eta_{\varepsilon}(t / s) f(z, s) d s / s,
$$

$f(z, s)$ being the value of $f(z) \in S_{0}(2, k)$ at $s$. Clearly

$$
m_{\varepsilon}(t)=\int_{0}^{\infty} \eta_{\varepsilon}(t / s) m(s) d s / s=f_{\varepsilon}(\delta, t) .
$$

We apply the Phragmen-Lindelöf principle to the function $z \rightarrow G_{z} f_{\varepsilon}$ with

$$
G_{z} f_{\varepsilon}(x)=|x|^{k+z} \int \varphi(r(\xi)) f_{\varepsilon}(z, R \rho(\xi)) e^{i x \xi} d \xi
$$

Observe that analogously to (a)

$$
\begin{aligned}
\left\|G_{z} f_{\varepsilon}\right\|_{2}^{2} & \leqslant \int\left|\left(1+|x|^{k+1}\right) \int \varphi(r(\xi)) f_{\varepsilon}(z, R \rho(\xi)) e^{i x \xi} d \xi\right|^{2} d x \\
& \leqslant c\left\|f_{\varepsilon}(z)\right\|_{2, k+1}^{2} \leqslant c(1+\varepsilon)^{2}\|f(z)\|_{2, k}^{2} .
\end{aligned}
$$

For the last step we used the integration by parts and $\left\|m_{\varepsilon}\right\|_{2, l} \leqslant C\|m\|_{2, l .}$. Hence, for fixed $\varepsilon>0$ the function $z \rightarrow G_{z} f_{\varepsilon}$ is $L^{2}$-valued and uniformly bounded for $z \in \bar{S}$.

Since $f$ is continuous on $\bar{S}$ and analytic in the interior of $\bar{S}$, one can show by estimates similar to those above that $z \rightarrow G_{z} f_{\varepsilon}$ is continuous and analytic with respect to the $L^{2}$-norm.

On the boundary for $j=0,1$

$$
\left\|G_{j+i y} f_{\varepsilon}\right\|_{2} \leqslant c\left\|f_{\varepsilon}(j+i y)\right\|_{2, k+j} \leqslant c\|f(j+i y)\|_{2, k+j} .
$$

Hence,

$$
\left\|G_{z} f_{\varepsilon}\right\|_{2} \leqslant c \max _{j=0,1} \max _{y \in \mathbf{R}}\|f(j+i y)\|_{2, k+j}=c\|f\|_{\mathscr{F}},
$$

$c$ independent of $\varepsilon$. By Lebesgue's Dominated Convergence Theorem and Fatou's Lemma

$$
\begin{aligned}
& \left.\left.\int|| x\right|^{\gamma} \int \varphi(r(\xi)) m(R \rho(\xi)) e^{i x \xi} d \xi\right|^{2} d x \\
& \quad \leqslant\left.\left.\liminf _{\varepsilon \rightarrow \infty} \int|| x\right|^{\gamma} \int \varphi(r(\xi)) m_{\varepsilon}(R \rho(\xi)) e^{i x \xi} d \xi\right|^{2} d x \\
& \quad=\liminf _{\varepsilon \rightarrow \infty}\left\|G_{\delta} f_{\varepsilon}\right\|_{2}^{2} \leqslant c\|f\|_{\mathscr{F}}^{2} .
\end{aligned}
$$

The interpolation property then gives the desired result since the norm of $m$ as an element of the complex interpolation space

$$
\left[S_{0}(2, k), S_{0}(2, k+1)\right]_{\delta}=S_{0}(2, \gamma)
$$

is equal to $\inf \left\{\|f\|_{\mathscr{F}}: f \in \mathscr{F}, f(\delta)=m\right\}$. 
Proof of Theorem 1. The restriction in Lemma 1 on $m$ to be infinitely differentiable and compactly supported away from the origin can be removed by density arguments as in $[13,6.2 .1$ and 2, p. 25]. On account of Lemma 2 the bilinear

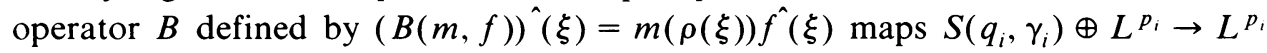
continuously for $q_{1}=2, \gamma_{1}=n / 2+\varepsilon, p_{1}=1+\varepsilon$, any small $0<\varepsilon<1 / 2$. Taking into account the embeddings $S(q, \alpha) \subset S(2, \alpha)$ if $2 \leqslant q \leqslant \infty$ and $S(q, \alpha+1 / 2) \subset S(2, \alpha)$ if $1<q<2$ [2, Theorem 5.3], we deduce that $B$ is also continuous for $q_{2}=1 / \varepsilon, \gamma_{2}=\gamma_{1}, p_{2}=p_{1}$ and $q_{3}=1+\varepsilon, \gamma_{3}=(n+1) / 2+\varepsilon$, $p_{3}=p_{1}$. On the other hand $S(q, \alpha) \subset L^{\infty}(0, \infty)$ if $1<q<\infty$ and $\alpha>1 / q[2$, Theorem 5.4] and $M_{2}\left(\mathbf{R}^{n}\right)=L^{\infty}\left(\mathbf{R}^{n}\right)$, hence $B$ is continuous for $q_{4}=1 / \varepsilon, \gamma_{4}=2 \varepsilon$, $p_{4}=2$ and $q_{5}=1+\varepsilon, \gamma_{5}=1, p_{5}=2$. The range $R$ in the $(1 / q, \gamma, 1 / p)$-diagram for which $B$ is continuous thus contains the five points $\left(1 / q_{i}, \gamma_{i}, 1 / p_{i}\right), i=1, \ldots, 5$, and by duality also the three points $\left(1 / q_{i}, \gamma_{i}, 1-1 / p_{i}\right), i=1,2,3$. By Connett and Schwartz [2, Theorem 5.5] $R$ is convex and since we may take $\varepsilon>0$ arbitrarily small $R$ contains the interior of the convex hull $H$ of the eight points $(1 / 2, n / 2,1)$, $(1 / 2, n / 2,0),(0, n / 2,1),(0, n / 2,0),(1,(n+1) / 2,1),(1,(n+1) / 2,0),(0,0,1 / 2)$, $(1,1,1 / 2)$. Note $S(q, \beta) \subset S(q, \alpha)$ for $\beta \geqslant \alpha$, thus for every $(1 / q, \alpha, 1 / p) \in H$ also the line $\{(1 / q, \beta, 1 / p): \beta \geqslant \alpha\}$ belongs to $R$. The region so obtained can be described by the two pairs of inequalities $\gamma>(n-1)|1 / p-1 / 2|+1 / q$ if $1 / q \geqslant$ $|1 / p-1 / 2|$ or $\gamma>n|1 / p-1 / 2|$ if $1 / q \leqslant|1 / p-1 / 2|, 1<p, q<\infty$. Finally, in the context of WBV-spaces, we can admit $q=1$ and $q=\infty$ without leaving $R$ since $\mathrm{WBV}_{1, \alpha+\varepsilon} \subset \mathrm{WBV}_{1+\varepsilon, \alpha}=S(1+\varepsilon, \alpha)$ if $\alpha \geqslant 1, \varepsilon>0$ and $\mathrm{WBV}_{\infty, \alpha} \subset \mathrm{WBV}_{q, \alpha}=$ $S(q, \alpha)$ if $1<q<\infty, \alpha>1 / q$. (See [5, Theorems 3 and 4].) This completes the proof of the theorem.

2. The above method of proof can be employed to give the following variant of Theorem 1:

THEOREM 2. Let $\rho$ be a $P$-homogeneous distance function with $P=\operatorname{diag}\left(\alpha_{1}, \ldots, \alpha_{n}\right)$. For each $j=1, \ldots, n$ and $\sigma \in\{0,1\}^{n}$ with $\sigma_{j}=0$ let $x_{j} \rightarrow D^{\sigma} \rho(x)$ be locally absolutely continuous for almost all $x^{j}=\left(x_{1}, \ldots, x_{j-1}, x_{j+1}, \ldots, x_{n}\right) \in \mathbf{R}^{n-1}$. For $\boldsymbol{\sigma}^{1}, \ldots, \sigma^{k} \in$ $\{0,1\}^{n}$ such that $\sigma^{1}+\cdots+\sigma^{k} \in\{0,1\}^{n}$ let $\prod_{j=1}^{k} D^{\sigma^{j}} \in L_{\text {loc }}^{s}\left(\mathbf{R}_{0}^{n}\right)$ for some $s$ with $2<s \leqslant \infty$. Now, if $1<p<\infty, 1 \leqslant q \leqslant \infty$ and $m \in \mathrm{WBV}_{q, \gamma}$ for some

$$
\gamma>n|1 / p-1 / 2|+(1 / q-(1-1 / s)|1 / p-1 / 2|)_{+},
$$

then $m \circ \rho \in M_{p}\left(\mathbf{R}^{n}\right)$ and $\|m \circ \rho\|_{M_{p}} \leqslant c\|m\|_{q, \gamma}$ with $c$ independent of $m$.

This result cannot be obtained from the general results of Madych [7], Fabes and Rivière [3], and Peral and Torchinsky [9]. It allows one to deal with nonsmooth distance functions $\rho$, e.g.

$$
\begin{gathered}
\rho_{4}(x)=\left|x_{1}\right|+\left(x_{1}^{2}+\left|x_{2}\right|\right)^{1 / 2}, \\
\rho_{5}(x)=\sum_{j=1}^{3}\left|x_{j}\right|^{\alpha_{1}}+\frac{1}{2} \operatorname{sgn}\left(x_{1}\right)\left|x_{1}\right|^{\beta_{1}}\left|x_{2}\right|^{\beta_{2}}, \quad \alpha_{j}, \beta_{k}>\frac{1}{2}, \beta_{1} / \alpha_{1}+\beta_{2} / \alpha_{2}=1 .
\end{gathered}
$$

Application of Theorem 2 to $\rho=\rho_{2}, \ldots, \rho_{5}$ yields

(i) $(1-\rho(\xi))_{+}^{\lambda} \in M_{p}\left(\mathbf{R}^{n}\right)$ if $\lambda>(n-1+1 / s)|1 / p-1 / 2|$ and $1<p<\infty$. 
In the different cases it suffices if $s>2$ satisfies $1 / s>\max \left(1-\alpha_{j}\right)$ for $\rho_{2}, s<5$ for $\rho_{3}, s=\infty$ for $\rho_{4}$, and $1 / s>\max \left(1-\alpha_{j}\right)$ as well as $1 / s>\max \left(1-\beta_{k}\right)$ for $\rho_{5}$.

(ii) $e^{i \rho(\xi)}(1+\rho(\xi))^{-\beta} \in M_{p}\left(\mathbf{R}^{n}\right)$ if $\beta>n|1 / p-1 / 2|$ and $1<p<\infty$.

The proof of Theorem 2 is analogous to that of Theorem 1 , therefore we only indicate the modifications: Instead of $g_{2}$ one considers

$$
g_{3}(f)(x)=\left(\int_{0}^{\infty} \int t^{-\nu}\left(\prod_{j=1}^{n}\left(1+t^{-\alpha_{t}}\left|x_{j}-y_{j}\right|\right)\right)^{-2 \kappa}\left|K_{t} * f(y)\right|^{2} d y d t / t\right)^{1 / 2}
$$

where $\kappa>1 / 2$. This $g$-function has the same $L^{p}$-behaviour as the $g_{2}$-function. Instead of Lemma 1 one proves

$$
\int\left|\left(\prod_{j=1}^{n}\left(1+\left|x_{j}\right|\right)\right)^{\kappa} F^{-1}[\varphi(r(\xi)) m(t \rho(\xi))](x)\right|^{2} d x \leqslant c\|m\|_{q, \gamma}^{2}
$$

if $\gamma=n \kappa, 1 / 2<\kappa \leqslant 1,1 / q=1 / 2-\kappa / s$. This can be done in the same manner as in the proof of Lemma 1 but interpolating between $\gamma=0$ and $\gamma=n$. In case $\gamma=n$ one has to apply Hölder's inequality to (1.1) to separate the terms depending on $m$ from those containing $\rho$ and its partial derivatives.

\section{REFERENCES}

1. O. V. Besov, V. P. $\mathrm{Il}^{\prime}$ in and P. I. Lizorkin, $L^{p}$-estimates of a certain class of nonisotropic singular integrals, Soviet Math. Dokl. 7 (1966), 1065-1069.

2. W. C. Connett and A. L. Schwartz, The theory of ultraspherical multipliers, Mem. Amer. Math. Soc. No. 183 (1977).

3. E. B. Fabes and N. M. Rivière, Singular integrals with mixed homogeneity, Studia Math. 27 (1966), 19-38

4. C. Fefferman, A note on spherical summation multipliers, Israel J. Math. 15 (1973), 44-52.

5. G. Gasper and W. Trebels, A characterization of localized Bessel potential spaces and applications to Jacobi and Hankel multipliers, Studia Math. 65 (1979), 243-278.

6. J. Löfström, Some theorems on interpolation spaces with applications to approximation in $L^{p}$, Math. Ann. 172 (1967), 176-196.

7. W. R. Madych, On Littlewood-Paley functions, Studia Math. 50 (1974), 43-63.

8. J. Peetre, Applications de la théorie des espaces d'interpolation dans l'analyse harmonique, Ricerche Mat. 15 (1966), 3-36.

9. J. Peral and A. Torchinsky, Multipliers in $H^{p}\left(\mathbf{R}^{n}\right), 0<p<\infty$. Ark. Mat. 17 (1979), 224-235.

10. N. M. Rivière, Singular integrals and multiplier operators, Ark. Mat. 9 (1971), 243-278.

11. S. Sjöstrand, On the Riesz means of the solution of the Schrödinger equation, Ann. Scuola Norm. Sup. Pisa Cl. Sci. (4) 24 (1970), 331-348.

12. E. M. Stein, Classes $H^{p}$ et multiplicateurs: Cas n-dimensionnel, C. R. Acad. Sci. Paris Sér. A-B 264 (1967), A107-A108.

13. Singular integrals and differentiability properties of functions, Princeton Univ. Press, Princeton, N. J., 1971.

14. E. M. Stein and S. Wainger, Problems in harmonic analysis related to the curvature, Bull. Amer. Math. Soc. (N. S.) 84 (1978), 1239-1295.

Fachbereich Mathematik, T. H. Darmstadt, D - 6100 Darmstadt, Federal Republic of Germany 